\title{
Belief-based and analytic processing in transitive inference depends on premise integration difficulty
}

\author{
GLENDA ANDREWS \\ Griffith University, Gold Coast Campus, Southport, Queensland, Australia
}

\begin{abstract}
Three experiments investigated belief-based versus analytic processing in transitive inference. Belief-based and analytic processing were inferred from conclusion acceptance rates for problems with conclusions that were either valid or invalid and believable or unbelievable. Premise integration difficulty was manipulated by varying premise integration time (Experiment 1), premise presentation order (Experiment 2), and the markedness of the relational terms in the premises (Experiment 3). In all the conditions, reasoning accuracy and rated confidence were lower on conflict problems, where belief-based and analytic processes yielded different responses. Participants relied more on analytic processing and less on belief-based processing in conditions in which premise integration was easier. Fluid intelligence and premise integration ability predicted analytical reasoning on conflict problems after reasoning on the no-conflict problems was controlled for. The findings were related to three dual-process models of belief bias. They provide the first evidence of belief bias in transitive inference.
\end{abstract}

Belief bias in reasoning is the tendency to draw or accept conclusions on the basis of one's beliefs, rather than on whether the conclusions follow logically from the premises (Oakhill \& Johnson-Laird, 1985). For example, given the premises The horse is larger than the cow and The cow is larger than the elephant, the conclusion The elephant is larger than the horse suggests the presence of belief bias, whereas The horse is larger than the elephant is the logically correct transitive inference.

Most accounts of belief bias invoke dual-process models of reasoning. According to these models, two types of cognitive processes underlie human reasoning. Nonanalytic processes are rapid, parallel, and automatic in their operation and are thought to include retrieval of beliefs and prior knowledge. Analytic processes permit abstract thinking, but they operate more slowly, are effortful, and impose demands on working memory and other fluid capacities (Evans, 2003). The two processes usually work together, but in some situations, they come into conflict. Dual-process models attribute belief bias to the dominance of belief-based processing over analytic processing.

There have been many demonstrations of belief bias in reasoning (see Evans, Newstead, \& Byrne, 1993, for a review). The findings are consistent with dual-process models. Other evidence for dual-process models is provided by neuroimaging studies showing that different neural pathways are activated during belief-based and analytic reasoning (Goel \& Dolan, 2003) and by findings that individuals with higher fluid intelligence are better able to resist belief bias (Stanovitch \& West, 2008) and that belief-based and analytic processing have different developmental trajectories (De Neys \& Van Gelder, 2009; Gilinsky \& Judd, 1994).

The present research examined belief-based and analytic processing in transitive inference, similar to the example above. No previous research appears to have addressed this issue. Three experiments tested the general hypothesis that individuals give more belief-based responses under conditions in which analytic processing is difficult.

Most previous research has examined belief bias in categorical syllogisms. Two premise statements are presented. Participants generate conclusions that follow from the premises, or they evaluate conclusions presented to them. The following is a valid syllogism with an unbelievable conclusion. No musicians are Italians. All barbers are musicians. Therefore, no barbers are Italians. Invalid conclusions are either determinately or indeterminately invalid. Determinately invalid conclusions are incompatible with all situations implied by the premises. Such conclusions cannot follow from the premises. Indeterminately invalid conclusions are compatible with some (not all) situations implied by the premises.

In the conclusion evaluation procedure, analytic and belief-based processing are inferred from acceptance rates for four problem types: valid believable (VB), valid unbelievable (VU), invalid believable (IB), and invalid unbelievable (IU). In VB and IU problems, belief-based and analytic processes yield the same conclusions. VU and IB problems are known as conflict problems, because belief-

G. Andrews, g.andrews@griffith.edu.au 
based and analytic processes yield conflicting responses. Research using categorical syllogisms has yielded three main findings. (1) Acceptance rates are higher for valid than for invalid problems. (2) Acceptance rates are higher for believable than for unbelievable conclusions. These effects are consistent with the use of analytic and beliefbased processing, respectively. (3) The believability effect is sometimes greater for invalid than for valid syllogisms. This interaction occurs with indeterminately invalid conclusions.

Specific models have been proposed to account for some or all of these findings. Stupple and Ball (2008) characterized existing dual-process models as either belief-first, reasoning-first, or parallel-process models, reflecting the hypothesized sequence in which beliefbased and analytic processing occur. Evans (2007) also identified three classes of models - namely, preemptive, parallel-competitive, and default interventionist models. In preemptive models, conflict between belief-based and analytic processing is avoided, because a decision is made at the outset of each problem as to whether responses will be determined by beliefs or analytic processes. The basis for this decision is some superficial aspect of the reasoning problem. In the parallel-competitive models, beliefbased and analytic processes proceed simultaneously, and each produces a response. When the two responses differ (VU, IB problems), the conflict must be resolved. Analytic processes can overrule the belief-based processes to some extent, but the greater speed of the belief-based processing means that it will sometimes precede and neutralize the responses of analytic processes. In the default interventionist model, shallow heuristic (belief-based) processes cue a default mental model that yields an initial response, which might subsequently be confirmed or revised by analytic processes. Evans (2007) showed that the three classes of models can be mapped to the same equation; therefore, distinguishing between them on the basis of acceptance rates alone is not straightforward. Including additional independent and dependent variables and procedural constraints might be helpful in this regard.

Evans and Curtis-Holmes (2005) examined the effect of time constraints on acceptance rates for VB, VU, IB, and IU categorical syllogisms. Conclusion evaluation time was manipulated, with premise-encoding time held constant. Participants in the free-time condition relied more on analytic reasoning and less on belief-based reasoning than did those in the rapid response condition, who were allowed just $5 \mathrm{sec}$ to read and evaluate the conclusion. The difference was due to lower acceptance of VU conclusions and higher acceptance of IB conclusions in the rapid response condition. The findings were interpreted in terms of the selective scrutiny model (Evans, Barston, \& Pollard, 1983; Evans et al., 1993). In this preemptive belief-first model, participants tend to accept believable conclusions. They are more likely to engage in analytic processing when conclusions are unbelievable. More time was available for analytic processing in the free-time condition; therefore, more VU conclusions were accepted in this condition. However, the findings do not preclude other accounts (Evans, 2007).
Quayle and Ball (2000) tested a reasoning-first model of belief bias. According to their metacognitive uncertainty model, people attempt to reason analytically, but they default to belief-based processing as a second-best option when the demands of the reasoning task exceed their working memory capacity. In Experiment 1, indeterminately invalid categorical syllogisms were more difficult and were evaluated with lower confidence than were valid syllogisms. This was attributed to the higher working memory load imposed by the invalid syllogisms. Experiment 3 examined more directly the role of working memory in the larger believability effect on invalid problems. The validity $\times$ believability interaction was present only for participants with low working memory capacity. These findings supported the metacognitive uncertainty model and the general notion that people resort to beliefbased processing when the demands of analytic processing exceed available capacity.

Stupple and Ball (2008) examined the effect of figural complexity on belief-based and analytical processing. They presented VB, VU, IB, and IU categorical syllogisms in two different figures. Figure refers to the order of the three classes in the premises. The standard effects of validity and believability and their interaction were observed for acceptance rates. The believability effect was larger for syllogisms with the more complex figure, in which premise integration is more difficult. Premise inspection times were longer for conflict (VU, IB) problems than for noconflict (VB, IU) problems. The findings were interpreted as providing strongest support for parallel-processing models. Sloman's (1996) parallel model includes associative (nonanalytic) and rule-based (analytic) processes, which produce different outputs on VU and IB problems. The resulting conflict takes time to resolve.

Roberts and Sykes (2003) examined belief bias in spatial and temporal relational inference problems, rather than in categorical syllogisms. Each problem consisted of four premises that defined the spatial or temporal relations among five entities and a conclusion that was believable or unbelievable and valid or invalid. The following is an example of a temporal problem whose conclusion is invalid and unbelievable for individuals familiar with world history. Elizabeth II reigned after Queen Victoria. Edward VII reigned after Elizabeth II. While Edward VII reigned, South Africa abolished apartheid. Lincoln abolished slavery during the reign of Queen Elizabeth. Therefore, Lincoln abolished slavery after South Africa abolished apartheid. Experiments 1 and 2 used one-model problems in which the premises allowed only one arrangement of the entities; therefore, invalid conclusions were determinately invalid. In Experiment 1, reasoning accuracy was higher with parallel presentation in which the premises and conclusion were available simultaneously than with serial presentation in which premises were presented one at a time and were removed when the conclusion appeared. Significant analytic processing occurred in both conditions but was stronger in the easier parallel condition than in the more difficult serial condition. Beliefbased processing was observed in the parallel condition, but not in the serial condition. Belief-based processing 
was more evident in Experiment 3, which employed twomodel problems in which two different arrangements of the entities were consistent with the premises. The interaction between validity and believability was present when indeterminately (but not determinately) invalid conclusions were analyzed. This replicated the pattern usually observed with categorical syllogisms. However, none of the models developed to account for belief bias in categorical syllogisms could account for all of Roberts and Sykes's findings. Some findings supported the view that participants resort to nonanalytic processes when analytic processing is difficult. However, although analytic responding was reduced, belief-based responding was not always observed in the more difficult conditions.

The present project extended belief bias research to transitive inference. The first aim was to determine the extent to which belief-based and analytic processing are used in transitive inference. No existing studies appear to have addressed this issue. To the extent that both types of processes are employed, significant effects of validity and believability should be observed on conclusion acceptance rates. To the extent that belief-based and analytic processes come into conflict during reasoning, VU and IB problems in which these processes conflict should be more difficult than VB and IU problems in which there is no such conflict. This would be evidenced by a significant validity $\times$ believability interaction when acceptance rates are expressed in terms of accuracy.

The second aim was to examine the effects on belief-based and analytic processing of experimental manipulations previously shown to influence the difficulty of transitive inference. In Experiment 1, premise-encoding/integration difficulty was manipulated by varying the time allowed for this to occur. Conclusion evaluation time was also manipulated. In Experiments 2 and 3, premise-encoding/integration difficulty was manipulated by varying the presentation order of the premises and the markedness of the relational terms in the premises, respectively. Previous research using categorical syllogisms suggests that there will be more beliefbased processing and less analytic reasoning in the more difficult conditions. However, Roberts and Sykes's (2003) research on relational inferences, which are more comparable than categorical syllogisms to transitive inferences, yielded mixed findings. The effects of these manipulations on conclusion acceptance rates and reasoning accuracy will be examined to determine whether conflict and no-conflict problems are differentially affected.

The third aim was to assess participants' awareness of the conflict between belief-based and analytic processes. Expressed levels of confidence might reflect this. If so, confidence should be higher for no-conflict problems than for conflict problems. The fourth aim was to examine two potential correlates of the ability to resolve conflict between belief-based and analytic processing. To address this issue, measures of fluid intelligence and premise integration ability were administered in Experiment 3.

In transitive inference problems, participants are given premises (e.g., $a>b, b>c$ ), and they evaluate or infer the relation between nonadjacent elements $(a>c)$. A concrete example with neutral content is the following: Tony is taller than Chris, Chris is taller than David, therefore, Tony is taller than David. Most people make transitive inferences by encoding the premises and integrating them by mentally arranging the elements into an ordered array, either from left to right or from top to bottom (Prado, Van der Henst, \& Noveck, 2008; Trabasso, Riley, \& Wilson, 1975). Having ordered the elements, Tony-Chris-David, they can easily conclude, by inspecting the array, that Tony is taller than David.

Evidence from a study that used a dual-task paradigm with probe reaction time as the secondary task (Maybery, Bain, \& Halford, 1986) suggested that premise encoding and premise integration impose different types of demand. The presence of negation (e.g., not above) and marked lexical terms (e.g., below) in the premises increased the time required to encode the premises but did not affect secondary task performance, whereas premise integration increased probe reaction time on the secondary task, relative to a task in which no integration was required. The processing load imposed by premise integration appears to occur because no element can be assigned to its position in the array on the basis of a single premise; rather, the premises must be considered jointly. For example, Tony is taller than Chris tells us that Tony is in either first or second position, but we can determine which only by considering the second premise. Halford, Wilson, and Phillips (1998) demonstrated that premise integration is a complex ternary-relational process. The two binary-relational premises $(a>b ; b>c)$ must be integrated to construct the ordered triple $(a>b>c)$, and this constitutes a ternary relation. Thus, premise-encoding and integration components of transitive inference tasks are more demanding than conclusion evaluation.

Research in cognitive development (e.g., Halford, 1984; Thayer \& Collyer, 1978) showed that three-term transitive inference problems are susceptible to a nonintegrative strategy that involves labeling the end elements. Element $a$ is labeled as large because it occurs only once in the premises as the larger element. Element $c$ is labeled as small, because it occurs only once in the premises as the smaller element. Labeling can be avoided by using five-term problems, $a>b>c>d>e$, with four premises, $a>b, b>c, c>d, d>e$, in which participants infer the relation between elements $b$ and $d$. Labeling is no longer an effective strategy, because elements $b$ and $d$ each occur in two premises and are labeled as larger in one premise and smaller in another premise (Andrews \& Halford, 1998; Halford, 1984). Thus, five-term problems were used in the present research.

Belief-based and analytic processing were examined using four types of problems (shown in Table 1) in which the conclusions varied in validity and believability. Participants evaluated each conclusion and rated their confidence in each decision. The four premises were always presented simultaneously and prior to the conclusion. This procedure differs from those in most existing studies, in which premises and conclusions are simultaneously available. One exception is the serial condition in Roberts and Sykes (2003, Experiment 1), where there was evidence of analytic processing but not belief-based processing. 
Table 1

Examples of the Four Problem Types

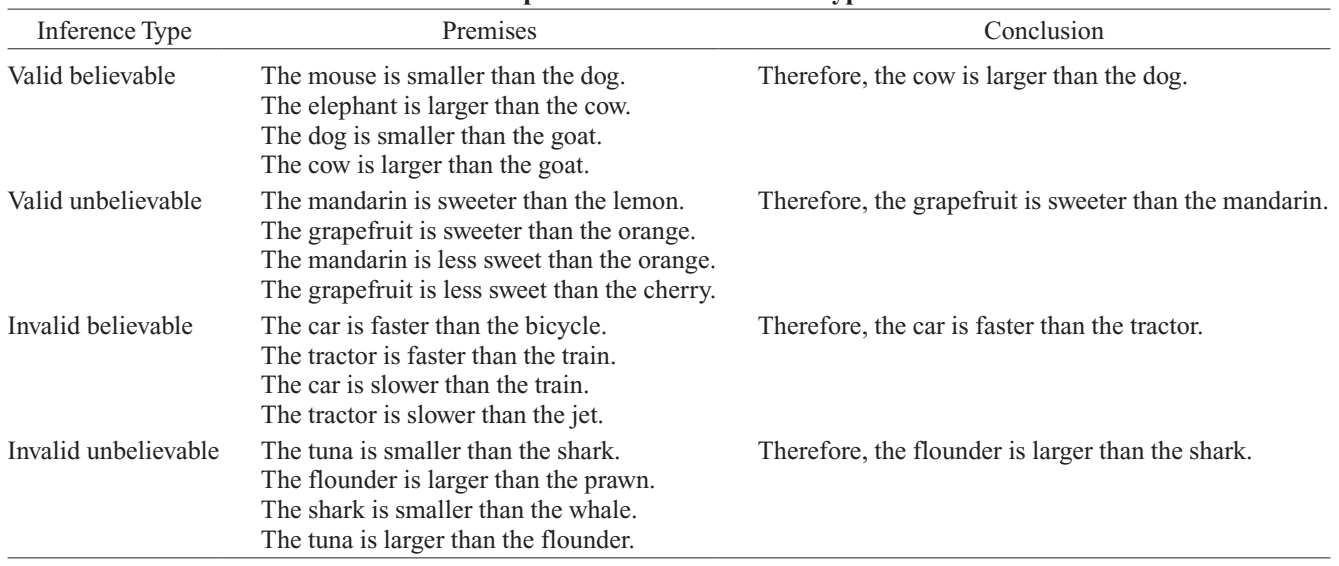

In VB and IU problems, the four premises were all believable, whereas in the VU and IB problems, two premises were believable and two were unbelievable. The unbelievable premises were unlikely, rather than definitionally false. For example, the IB problem in Table 1 includes the unbelievable premise The tractor is faster than the train. Although trains generally travel faster than tractors, this might not be true in every situation. Unbelievable conclusions were also empirically unlikely (e.g., The grapefruit is sweeter than the mandarin). Definitionally false statements (e.g., Some cats are not animals), which are included in the categorical syllogisms in some studies (e.g., Klauer, Musch, \& Naumer, 2000; Stupple \& Ball, 2008), yield larger believability effects.

The transitive inference problems were one-model problems in which only one arrangement of the elements was compatible with the premises. Invalid conclusions were determinately invalid in the present research, whereas in the studies of belief bias in categorical syllogisms reviewed above, they were indeterminately invalid.

Although the research was not specifically designed to distinguish between specific models, the findings will be considered in relation to reasoning-first, parallel-process, default interventionist models. Preemptive models in which conclusion believability triggers belief-based processing will not be considered, because the conclusions and premises were not simultaneously available in the present procedure. Delaying processing of the premises until the conclusions were presented would not be a workable strategy.

Parallel-process models would provide a ready and natural explanation of lower accuracy and lower confidence for conflict (VU, IB) than for no-conflict (VB, IU) problems because conflict occurs only when both processes are applied. In the default interventionist model, individuals who make conscious efforts to inhibit their default beliefbased responses might realize the conflict (Evans, 2007). However, this is not guaranteed, because analytic processing does not always occur. In reasoning-first models, individuals will first attempt to use analytic processing. When this fails, they will default to belief-based processing, which yields correct responses on no-conflict problems and incorrect responses on conflict problems.

\section{EXPERIMENT 1}

Experiment 1 extended Evans and Curtis-Holmes's (2005) research by examining the effects of premiseencoding/integration time and conclusion evaluation time on belief-based and analytic processing in transitive inference. Higher acceptance rates were expected for valid than for invalid conclusions and for believable than for unbelievable conclusions. On the basis of research showing that premise integration is more demanding than conclusion evaluation (Maybery et al., 1986), belief-based and analytic processing were expected to be more sensitive to premise-encoding/integration time than to conclusion evaluation time. More belief-based processing and less analytic processing were expected in the shorter premiseencoding/integration condition.

Accuracy and confidence were expected to be higher for no-conflict (VB, IU) problems than for conflict (VU, IB) problems. There are two potential loci of the conflict in VU and IB problems. The conflict might occur during premise integration when unbelievable premises conflict with prior beliefs and make premise integration more difficult (Thompson, 1996). If so, conflict problems should be more sensitive than no-conflict problems to premiseencoding/integration time. Conflict might also occur during conclusion evaluation when the VU and IB conclusions are consistent either with prior beliefs or with analytic processing but not with both. If so, conflict problems should be more sensitive than no-conflict problems to conclusion evaluation time.

\section{Method}

Participants. The participants were 104 students (19 of them male, 85 female) enrolled in undergraduate psychology courses. Their mean age was 24.26 years $(S D=8.27)$. All were native speakers of English. Twenty-six participants were assigned to each 
of four conditions reflecting combinations of two premise-encoding/ integration times (10 and $20 \mathrm{sec}$ ) and two conclusion evaluation times ( 5 and $10 \mathrm{sec}$ ). There were no significant differences in age or gender balance across the conditions.

Stimuli and Procedure. Each transitive inference problem had four premises that, when integrated, yielded an ordered five-term sequence of the form $a>b>c>d>e$. The elements in each problem were drawn from the same category (e.g., animals, vehicles, household items). Transitive relations (e.g., taller-shorter, heavier-lighter, faster-slower) related the elements in the premises and conclusions. Unmarked (e.g., taller) and marked (e.g., shorter) forms were each used twice in each premise set. Valid conclusions were stated in the form $b>d$. Invalid conclusions were stated in the form $d>b$ and were always determinately invalid. Four problem types were generated by varying conclusion validity and believability. There were 16 test problems, 4 of each type (VB, VU, IB, IU), and 2 practice problems with the same form as the test problems but with neutral content. Examples of the test problems are shown in Table 1.

Group administration was used. Problems were presented using PowerPoint software running on a laptop computer and a data projector to display the screen images on a large white screen. Instructions were displayed on the screen and read aloud by the experimenter. The participants were told that they should assume that the premises were true. Even if they did not believe that the premises were always true, they should respond as if they were always true. They were instructed to read the premises carefully and to think about how each premise related to the others. When the conclusion appeared, they were to read and evaluate it in terms of the premises, then record their yes/no response and their confidence in their decision on the sheet provided. Confidence was rated on a 3 -point scale $(0=l o w$; $1=$ medium $; 2=$ high .

Two practice problems were presented. A ready signal was displayed for $3 \mathrm{sec}$. Then the premises were displayed simultaneously for $30 \mathrm{sec}$, in a quasi-random top-down order. The premise $a>b$ never appeared in top position. The conclusion was then presented for $10 \mathrm{sec}$, followed by a respond now signal for $10 \mathrm{sec}$, during which the participants recorded their responses. Feedback was provided after each practice problem. The premises from the preceding problem, the corresponding five-term sequence (expressed in the form $a>b>c>d>e$, but using the elements from the problem), and the conclusion were displayed on the same slide, along with a statement indicating the validity of the conclusion. The instructions and feedback were intended to encourage integration of the premises into an ordered series.

The participants were then informed of the specific durations they would experience in the test problems. These differed according to the condition to which they were assigned. These durations were specified in the PowerPoint presentations, and timing was controlled by this software. No feedback was provided on test problems. Otherwise, the procedure was identical to that for the practice problems. The 16 test problems were arranged into four 4-problem blocks, each containing one problem of each type (VB, VU, IB, IU). A 30- sec break was inserted after each block. The top-down order of the premises did not differ across problem types.

Following the reasoning task, 91 participants rated the plausibility of the conclusions of the test problems (presented alone) using a 7 -point scale $(1=$ extremely implausible to $7=$ extremely plausible).

\section{Results and Discussion}

Manipulation check. A repeated measures $t$ test confirmed that believable conclusions $(M=6.22, S E=$ 0.068 ) were significantly more plausible than unbelievable conclusions $(M=2.01, S E=0.059)[t(90)=43.18$, $p<.001]$.

Acceptance rates. Acceptance rates for VU, IB, and IU items were each based on four items. VB acceptance rates were based on three items, due to a wording error in one item. The acceptance rates (proportion of yes responses) are shown in Table 2 . A mixed 2 (validity) $\times 2$ (believability) $\times 2$ (premise-encoding time) $\times 2$ (conclusion evaluation time) ANOVA revealed significant main effects of validity $\left[F(1,100)=328.98, p<.001, \eta^{2}=.767\right]$ and of believability $\left[F(1,100)=83.85, p<.001, \eta^{2}=.456\right]$. Acceptance rates were higher for valid $(M=.75, S E=$ $.015)$ than for invalid $(M=.26, S E=.016)$ problems and for believable $(M=.62, S E=.015)$ than for unbelievable $(M=.39, S E=.015)$ problems. The nonsignificant validity $\times$ believability interaction confirmed that the believability effect did not differ significantly for valid (.194) versus invalid (.252) problems, and the validity effect did not differ for believable (.456) versus unbelievable (.514) problems. There was no significant main effect of conclusion evaluation time and no significant interactions of this variable with validity or believability (largest $F=1.23$ ).

There was a significant validity $\times$ premise-encoding time interaction $\left[F(1,100)=26.28, p<.001, \eta^{2}=.208\right]$. The validity effect was larger in the 20 -sec condition $\left[F(1,51)=242.68, p<.001, \eta^{2}=.826\right]$ than in the 10 sec condition $\left[F(1,51)=93.31, p<.001, \eta^{2}=.647\right]$, indicating more analytic processing when more time was available. There was also a significant believability $\times$ premise-encoding time interaction $[F(1,100)=7.66, p<$ $\left..001, \eta^{2}=.071\right]$. The believability effect was somewhat larger in the 10-sec condition $[F(1,51)=49.86, p<.001$, $\left.\eta^{2}=.494\right]$ than in the 20 -sec condition $[F(1,51)=36.83$, $\left.p<.001, \eta^{2}=.419\right]$, indicating less belief-based processing when more time was available.

Table 2

Acceptance and Confidence Rates by Conclusion Type and Premise-Encoding Time (Experiment 1)

\begin{tabular}{|c|c|c|c|c|c|c|c|c|}
\hline \multirow[b]{4}{*}{ Conclusion Type } & \multicolumn{8}{|c|}{ Premise-Encoding Time } \\
\hline & \multicolumn{4}{|c|}{$\begin{array}{l}\text { Acceptance Rate } \\
\text { (Proportion) }\end{array}$} & \multicolumn{4}{|c|}{ Confidence Rating } \\
\hline & \multicolumn{2}{|c|}{$10 \mathrm{sec}$} & \multicolumn{2}{|c|}{$20 \mathrm{sec}$} & \multicolumn{2}{|c|}{$10 \mathrm{sec}$} & \multicolumn{2}{|c|}{$20 \mathrm{sec}$} \\
\hline & $M$ & $\overline{S E}$ & $M$ & $\overline{S E}$ & $M$ & $S E$ & $M$ & $S E$ \\
\hline Valid believable & .82 & .03 & .87 & .03 & 1.12 & 0.06 & 1.52 & 0.06 \\
\hline Valid unbelievable & .57 & .04 & .74 & .04 & 0.76 & 0.06 & 1.08 & 0.06 \\
\hline Invalid believable & .51 & .04 & .27 & .04 & 0.86 & 0.06 & 1.21 & 0.06 \\
\hline Invalid unbelievable & .18 & .03 & .09 & .03 & 0.94 & 0.08 & 1.46 & 0.08 \\
\hline
\end{tabular}


Specifically, significantly more VU conclusions were accepted in the 20 -sec than in the 10 -sec condition $\left[F(1,102)=11.66, p=.001, \eta^{2}=.103\right]$, whereas significantly fewer IB $\left[F(1,102)=20.167, p<.001, \eta^{2}=\right.$ $.167]$ and IU $\left[F(1,102)=6.29, p=.014, \eta^{2}=.058\right]$ conclusions were accepted in the 20 -sec than in the 10 -sec condition. Acceptance of VB conclusions in the 10- and 20 -sec conditions did not differ significantly. Thus, conflict problems were more sensitive than no-conflict problems to premise-encoding/integration time.

This was confirmed when accuracy (acceptance of VB and VU, rejection of IB and IU conclusions) was the dependent measure. A mixed 2 (validity) $\times 2$ (believability) $\times 2$ (premise-encoding time) ANOVA revealed a significant main effect of premise-encoding/integration time $\left[F(1,102)=25.99, p<.001, \eta^{2}=.203\right]$, which was modified by a significant validity $\times$ believability interaction $\left[F(1,102)=84.68, p<.001, \eta^{2}=.454\right]$ and a significant validity $\times$ believability $\times$ premise-encoding/ integration time interaction $[F(1,102)=7.73, p=.006$, $\left.\eta^{2}=.07\right]$. The two-way interaction indicated that conflict (VU, IB) problems were more difficult than no-conflict (VB, IU) problems. This interaction was stronger in the 10 -sec condition $\left[F(1,51)=49.86, p<.001, \eta^{2}=.494\right]$ than in the $20-\sec$ condition $[F(1,51)=36.83, p<.001$, $\left.\eta^{2}=.419\right]$.

That reasoning was influenced by premise-encoding time, but not by conclusion evaluation time, is consistent with transitive inference research showing that integrating the premises is the most demanding component of transitive inference (Maybery et al., 1986). Conclusion generation or evaluation is quite straightforward once integration has occurred.

The interactions show that the locus of the conflict between belief-based and analytic processes was at premise integration, rather than at conclusion evaluation. Detection of conflict during premise integration is clearly consistent with parallel-process models because belief-based and analytic processes operate from the outset and the conflict would be apparent when unbelievable premises were encountered. The 20 -sec condition would allow more time to resolve the conflict in favor of analytic processing. In the default interventionist model, an initial mental model would be generated using belief-based processes. For the VU problem (Table 1), for example, this might consist of the fruits named in the premises and prior beliefs about their relative sweetness. If no analytic processing occurs, the initial model would be retained, no conflict would be experienced, and the conclusion would be rejected. This would occur more frequently in the 10 -sec than in the 20 -sec condition, which would allow more time for analytic processing to inhibit beliefs. In reasoning-first models, belief-based responses are made when analytic processing fails. The shorter premise integration time increased the difficulty of premise integration, so default to belief-based responses would be more likely in the 10 -sec condition. The effect would be evident on conflict problems.

Confidence ratings. The mean confidence ratings are shown in Table 2 . A mixed 2 (validity) $\times 2$ (believabil- ity) $\times 2$ (premise-encoding time) $\times 2$ (conclusion evaluation time) ANOVA revealed a significant main effect of believability $\left[F(1,100)=16.67, p<.001, \eta^{2}=.143\right]$. Confidence was higher for believable $(M=1.18, S E=$ $0.037)$ than for unbelievable $(M=1.06, S E=0.043)$ problems. The main effect of believability was modified by a significant validity $\times$ believability interaction $\left[F(1,100)=75.50, p<.001, \eta^{2}=.430\right]$. Confidence was lower for conflict problems $(M=0.98, S E=0.039)$ than for no-conflict problems $(M=1.26, S E=0.042)$. This suggests that participants were aware of the conflict in VU and IB problems when they rated their confidence in their evaluations. This is consistent with all the models. The clear parallel between confidence ratings and reasoning accuracy suggests that the participants were aware of the adequacy of the reasoning (cf. Quayle \& Ball, 2000).

Confidence was significantly higher in the $20-\mathrm{sec}$ $(M=1.32, S E=0.053)$ than in the $10-\sec (M=0.92$, $S E=0.053)$ premise-encoding/integration condition $\left[F(1,100)=27.68, p<.001, \eta^{2}=.217\right]$. Conclusion time had no significant effect on confidence and did not enter into any significant interactions [largest $F(1,100)=2.67$, $p=.11]$. The marginally significant validity $\times$ believability $\times$ premise-encoding time interaction $[F(1,100)=$ 3.83, $\left.p=.053, \eta^{2}=.037\right]$ showed that the difference between conflict and no-conflict problems was more pronounced in the 20 -sec than in the 10 -sec condition. Having extra time for premise encoding and integration increased confidence, as in Shynkaruk and Thompson (2006), and produced greater awareness of the difference between conflict and no-conflict problems.

In summary, the patterns of acceptance and confidence rates suggest that belief-based and analytical processes were employed in transitive inference. The participants experienced difficulty and were less confident when these processes conflicted. The locus of the conflict was premise integration, rather than conclusion evaluation. More belief-based processing and less analytical processing occurred when premise integration time was shorter. Conflict problems were more affected by premise integration time than were no-conflict problems.

\section{EXPERIMENT 2}

In Experiment 1, premises were always presented in scrambled order. Premise integration under such conditions has been analyzed as involving ternary-relational processing (Halford et al., 1998). This level of complexity makes transitive inference very difficult for children under 5 years of age (Andrews \& Halford, 1998, 2002; Halford, 1984) and for adults with frontal lobe impairments due to the degenerative effects of normal aging (Andrews \& Todd, 2008; Viskontas, Holyoak, \& Knowlton, 2005) and dementia (Waltz et al., 2004; Waltz et al., 1999). In Experiment 2 , integration complexity was manipulated by contrasting the scrambled order with a condition in which the order of the premises was consistent with the order of the elements in the five-term series - that is, $a>b, b>c$, $c>d, d>e$. In the consistent order condition, a chaining or concatenation strategy can be employed whereby each 
new element can be added to the chain as it is encountered. Thus, $a>b$ becomes $a>b>c$, which then becomes $a>b>c>d$, which then becomes $a>b>c>d>e$ (Andrews \& Halford, 1998; Waltz et al., 1999). The chaining strategy is binary relational because just one premise needs to be considered at a time. More analytic processing and less belief-based processing were expected in the consistent order condition.

Another modification was the inclusion of two problem sets. A new problem set was generated by reversing the conclusions of the original items as in Roberts and Sykes (2003). For example, the valid, believable conclusion The cow is larger than the dog became The dog is larger than the cow, which (given the premises in Table 1) is invalid and unbelievable. Thus, the problems in both sets had the same premises but different conclusions. The VB, VU, IB, and IU items in the original problem set became IU, IB, VU, and VB items, respectively, in the new problem set. This avoids confounding premise content with validity and believability (Thompson, 1996). However, it is still the case that conflict problems in both sets had some unbelievable premises, whereas the no-conflict problems did not.

\section{Method}

Participants. The sample consisted of 100 students (17 of them male, 83 female) who were enrolled in undergraduate psychology courses. Their mean age was 24.99 years $(S D=8.18)$. All were native speakers of English. There were 25 participants in each of the four conditions, reflecting the combination of two problem sets and two premise presentation orders.

Stimuli and Procedure. In each set, there were 16 test problems, 4 of each type (VB, VU, IB, and IU). In the scrambled condition, the premises were presented in scrambled order (as in Experiment 1). In the consistent condition, the top-down order of the premises was $a-b, b-c, c-d, d-e$. The order of the premises in the practice problems matched that in the test problems in each condition. Premiseencoding/integration time and conclusion evaluation time were held constant at 20 and $5 \mathrm{sec}$, respectively. The participants who received the original problem set rated the plausibility of the conclusions from the new problem set, whereas those who received the new problem set rated the plausibility of the conclusions from the original problem set.

\section{Results and Discussion}

Manipulation check. Believable conclusions $(M=$ $6.18, S E=0.106)$ were more plausible than unbelievable conclusions $(M=1.71, S E=0.096)$ in the original prob- lem set $[t(49)=24.88, p<.001]$. Believable conclusions $(M=5.86, S E=0.109)$ were more plausible than unbelievable conclusions $(M=2.19, S E=0.114)$ in the new problem set $[t(49)=22.04, p<.001]$.

Acceptance rates. Preliminary analyses revealed no significant effect or interactions involving problem set, so this variable was dropped from subsequent analyses. Acceptance rates are shown in Table 3. A mixed 2 (validity) $\times 2$ (believability) $\times 2$ (premise order) ANOVA revealed significant main effects of validity $[F(1,98)=$ 518.96, $\left.p<.001, \eta^{2}=.841\right]$ and believability $[F(1,98)=$ $\left.56.67, p<.001, \eta^{2}=.366\right]$. Acceptance rates were higher for valid $(M=.83, S E=.017)$ than for invalid $(M=$ $.17, S E=.016)$ problems and for believable $(M=.58$, $S E=.013)$ than for unbelievable $(M=.42, S E=.014)$ problems.

These main effects were modified by a significant validity $\times$ premise order interaction $[F(1,98)=11.88$, $\left.p<.001, \eta^{2}=.108\right]$ and a significant believability $\times$ premise order interaction $[F(1,98)=4.40, p=.039$, $\left.\eta^{2}=.043\right]$. The validity effect was stronger in the consistent $\left[F(1,49)=393.95, p<.001, \eta^{2}=.889\right]$ than in the scrambled $\left[F(1,49)=165.85, p<.001, \eta^{2}=.772\right]$ condition. The believability effect was stronger in the scrambled $\left[F(1,49)=51.45, p<.001, \eta^{2}=.512\right]$ than in the consistent $\left[F(1,49)=13.41, p=.001, \eta^{2}=.215\right]$ condition. Reducing the complexity of premise integration (consistent order condition) resulted in more analytic and less belief-based processing, relative to the scrambled condition.

These two-way interactions were modified by a validity $\times$ believability $\times$ premise order interaction $[F(1,98)=$ $\left.3.94, p=.05, \eta^{2}=.039\right]$. The main effects of validity and believability were significant in both conditions. The validity $\times$ believability interaction was significant only in the scrambled condition $\left[F(1,49)=4.66, p=.036, \eta^{2}=\right.$ $.036]$, where the difference between believable and unbelievable problems was greater for invalid (.277) than for valid $(.152)$ problems. These values $(.115, .127)$ did not differ significantly in the consistent condition.

The interactions showed that premise order had differential effects on the four problem types. Acceptance of VB conclusions $\left[F(1,98)=7.08, p=.009, \eta^{2}=.067\right]$ and VU conclusions $\left[F(1,98)=4.57, p=.035, \eta^{2}=\right.$ $.045]$ was significantly higher in the consistent than in the scrambled condition. Acceptance of IB conclusions was

Table 3

Acceptance and Confidence Rates by Conclusion Type and Premise Presentation Order (Experiment 2)

\begin{tabular}{|c|c|c|c|c|c|c|c|c|}
\hline \multirow[b]{4}{*}{ Conclusion Type } & \multicolumn{8}{|c|}{ Premise Order } \\
\hline & \multicolumn{4}{|c|}{$\begin{array}{c}\text { Acceptance Rate } \\
\text { (Proportion) }\end{array}$} & \multicolumn{4}{|c|}{ Confidence Rating } \\
\hline & \multicolumn{2}{|c|}{ Scrambled } & \multicolumn{2}{|c|}{ Consistent } & \multicolumn{2}{|c|}{ Scrambled } & \multicolumn{2}{|c|}{ Consistent } \\
\hline & $M$ & $S E$ & $M$ & $S E$ & $M$ & $S E$ & $M$ & $S E$ \\
\hline Valid believable & .86 & .02 & .94 & .02 & 1.25 & 0.07 & 1.68 & 0.07 \\
\hline Valid unbelievable & .70 & .04 & .82 & .04 & 0.92 & 0.07 & 1.41 & 0.07 \\
\hline Invalid believable & .36 & .04 & .18 & .04 & 1.01 & 0.08 & 1.24 & 0.08 \\
\hline Invalid unbelievable & .08 & .02 & .06 & .02 & 1.33 & 0.07 & 1.57 & 0.07 \\
\hline
\end{tabular}


significantly higher in the scrambled than in the consistent condition $\left[F(1,98)=11.50, p=.001, \eta^{2}=.105\right]$. Acceptance of IU conclusions was not significantly affected by premise order. Conflict problems were more sensitive than no-conflict problems to premise integration complexity.

This was confirmed when accuracy was the dependent measure. A mixed 2 (validity) $\times 2$ (believability) $\times$ 2 (premise order) ANOVA revealed a significant main effect of premise order $\left[F(1,98)=11.88, p<.001, \eta^{2}=\right.$ $.108]$, a significant validity $\times$ believability interaction $\left[F(1,98)=56.67, p<.001, \eta^{2}=.366\right]$, and a significant believability $\times$ premise order interaction $[F(1,98)=3.94$, $\left.p=.05, \eta^{2}=.039\right]$, which were all modified by a significant validity $\times$ believability $\times$ premise order interaction $\left[F(1,98)=4.40, p=.039, \eta^{2}=.043\right]$. The validity $\times$ believability interaction indicated that conflict (VU, IB) problems were more difficult than no-conflict (VB, IU) problems. This interaction was stronger when premise order was scrambled $\left[F(1,49)=51.54, p<.001, \eta^{2}=\right.$ $.512]$ than when it was consistent $[F(1,49)=13.41, p<$ $\left..001, \eta^{2}=.215\right]$.

The effects of premise presentation order on beliefbased and analytic processing are clearly consistent with reasoning-first models. Analytic processing is less likely to fail when premise integration complexity is reduced (consistent condition); therefore, there will be less need to default to belief-based processing. The effects are also consistent with parallel-process models, because consistent order of the premises would make it easier for analytic processes to dominate belief-based processes. The situation in relation to default interventionist models is less clear. However, to the extent that analytic processing occurs, it would likely be more successful in the consistent than in the scrambled condition.

Confidence ratings. Table 3 shows the mean confidence ratings. A mixed 2 (validity) $\times 2$ (believability) $\times$ 2 (premise order) ANOVA revealed a significant validity $\times$ believability interaction $[F(1,98)=61.16, p<.001$, $\left.\eta^{2}=.384\right]$. Confidence was lower for conflict $(M=1.15$, $S E=0.049)$ than for no-conflict $(M=1.45, S E=0.044)$ problems. Confidence was higher in the consistent $(M=$ $1.47, S E=0.06)$ than in the scrambled $(M=1.13, S E=$ $0.06)$ condition $\left[F(1,98)=16.86, p<.001, \eta^{2}=.147\right]$. There was also a significant validity $\times$ premise order interaction $\left[F(1,98)=14.68, p<.001, \eta^{2}=.13\right]$. The difference between premise order conditions was significant for all problem types [VB, $F(1,98)=18.91, p<.001$, $\eta^{2}=.162 ; \mathrm{VU}, F(1,98)=22.22, p<.001, \eta^{2}=.185 ; \mathrm{IB}$ $F(1,98)=4.83, p=.03, \eta^{2}=.047$; IU, $F(1,98)=5.53$, $\left.p=.021, \eta^{2}=.053\right]$, but the effect was greater for valid than for invalid problems.

Experiment 2 replicated the main findings of Experiment 1 . Acceptance and confidence rates suggested that belief-based and analytical processes were employed in transitive inference. The participants experienced difficulty and were less confident when these processes conflicted. Less belief-based processing and more analytic processing occurred when premise integration complexity was reduced by presenting the premises in consistent, rather than scrambled, order.

\section{EXPERIMENT 3}

Experiment 3 examined another factor that potentially affects the difficulty of premise encoding and integration-namely, lexical markedness. Premises that use marked forms of the relational expression (e.g., Tom is shorter than Mary) are known to take longer to encode than those with the corresponding unmarked (e.g., Mary is taller than Tom) forms (Evans et al., 1993), presumably because marked forms are first converted to the unmarked form (Maybery et al., 1986). For example, for the VB problem in Table 1, the premises The mouse is smaller than the dog and The dog is smaller than the goat would be converted to The dog is larger than the mouse and The goat is larger than the dog, respectively, prior to integration with the other premises. Given that the time available for premise encoding and integration was limited, use of unmarked forms only should reduce premise-encoding time and leave more time available for premise integration. If so, premise integration should be facilitated in the unmarked-only condition, and this should result in more analytic and less belief-based processing than in the mixed markedness condition.

Most theorists agree that analytic reasoning is related to general intelligence, whereas nonanalytic processing is not. According to the default interventionist model, higher ability individuals are more likely to engage analytic processes (Evans, 2007). According to reasoningfirst models, initial attempts at analytic processing would be more successful in higher ability individuals, so default to belief-based processing would occur less often (Quayle $\&$ Ball, 2000). According to parallel-process models (Sloman, 1996), analytic processes would be better able to dominate belief-based processes in higher than in lower ability individuals.

Two potential correlates of the capacity to resolve conflict between belief-based and analytic processes were examined. These were fluid intelligence and premise integration ability. According to Stanovitch and West (2008), associations with fluid intelligence are more likely when the reasoning task involves a conflict. The VU and IB problems meet this requirement. Stanovitch and West suggested that tests of fluid intelligence directly tap the analytic ability required to detect and resolve such conflict.

To the extent that accuracy on conflict problems depends on successful premise encoding and integration, we might expect individual differences in premise integration ability to predict reasoning accuracy. This ability was assessed in a subset of the sample using the $n$-term premise integration task (Andrews, Birney, \& Halford, 2006). Participants mentally constructed sequences of three, four, or five letters, on the basis of premises. The relations depicted in the premises were arbitrary (e.g., $Z>T$ ) and bore no relation to prior beliefs; therefore, the task could not be performed using belief-based processes.

Fluid ability and $n$-term scores should each predict accuracy on conflict problems, which require analytic processing. This should remain so after controlling for accuracy on the no-conflict problems, which could be performed using either belief-based or analytic processing. 


\section{Method}

Participants. The sample consisted of 152 students ( 38 of them male, 114 female) enrolled in undergraduate psychology courses. Their mean age was 21.54 years $(S D=7.04)$. Most $(96 \%)$ were native speakers of English. There were 38 participants in each of the four conditions, reflecting the combinations of two problem sets and two markedness conditions. A subgroup of 58 participants $(22$ from the unmarked-only and 36 from the mixed markedness conditions) completed the $n$-term premise integration task in a second session.

Stimuli and Procedure. The premises were always presented in scrambled order. Premise-encoding time and conclusion evaluation time were held constant at 20 and $5 \mathrm{sec}$, respectively. In the mixed markedness condition, both marked (e.g., shorter than, smaller than) and unmarked (e.g., taller than, larger than) forms were used in the premises (as in Experiments 1 and 2). In the unmarked-only condition, only the unmarked forms were used. The markedness of the relational terms in the practice problems matched the test problems in each condition.

Fluid intelligence was assessed using the Culture Fair Test, Scale 2, Form A (Cattell \& Cattell, 1973). Four subtests were administered using the standard instructions and applying the specified time limits. The maximum total score was 46.

The $n$-term task (Andrews et al., 2006) required the participants to use the premises to construct an ordered series of three, four, or five alphabetic letters. Examples are shown in Figure 1. There were three, four, and five premises for the three-term, four-term, and five-term items, respectively. One premise in each set consisted of a nonadjacent pair of letters, and the remaining premises related adjacent letters. A combination of $<$ and $>$ symbols was used in the premises, which were displayed in random top-down order on the left side of the screen. They remained visible until the participants had typed the required number of letters. On the right side was a row of three, four, or five boxes with $>$ signs between them. At the outset of each item, the leftmost box was highlighted in white. The first letter typed appeared here and remained in view momentarily before being replaced by an asterisk. Subsequent boxes were highlighted only when a valid letter (one that appeared in the premises) was typed in the preceding box. When the final letter had been entered, the entire sequence of letters was displayed briefly. The participants were advised to construct the entire sequence mentally before beginning to type, because they were unable to reorder the letters once they had been entered. No time limit was imposed. The items were blocked according to series length. There was one practice item and eight test items in each block. The maximum total score was 24 .

\section{Results and Discussion}

Acceptance rates. Preliminary analyses revealed no significant main effect or interactions involving problem set, so this variable was dropped from subsequent analyses. Acceptance rates are shown in Table 4. A mixed 2 (validity) $\times 2$ (believability) $\times 2$ (markedness) ANOVA revealed significant main effects of validity $[F(1,150)=$ 749.94, $\left.p<.001, \eta^{2}=.833\right]$ and of believability $\left[F(1,150)=94.97, p<.001, \eta^{2}=.387\right]$. Acceptance rates were higher for valid $(M=.828, S E=.013)$ than
Premises

$$
\begin{aligned}
& \mathrm{T}<\mathrm{P} \\
& \mathrm{P}>\mathrm{V} \\
& \mathrm{T}<\mathrm{V} \\
& \mathrm{B}>\mathrm{A} \\
& \mathrm{A}<\mathrm{F} \\
& \mathrm{F}>\mathrm{B} \\
& \mathrm{X}<\mathrm{A} \\
& \mathrm{N}<\mathrm{G} \\
& \mathrm{D}>\mathrm{G} \\
& \mathrm{N}>\mathrm{Q} \\
& \mathrm{L}<\mathrm{Q} \\
& \mathrm{G}>\mathrm{L}
\end{aligned}
$$

Correct Sequences

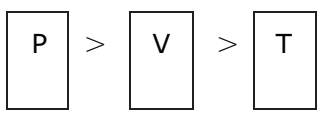

3-Term

4-Term

Figure 1. Example items at three levels of complexity in the $n$-term task. Premise information is shown on the left, and completed sequences are on the right. Based on Andrews, Birney, and Halford (2006). 
Table 4

Acceptance and Confidence Rates by Conclusion Type and Markedness Condition (Experiment 3)

\begin{tabular}{|c|c|c|c|c|c|c|c|c|}
\hline \multirow[b]{4}{*}{ Conclusion Type } & \multicolumn{8}{|c|}{ Markedness } \\
\hline & \multicolumn{4}{|c|}{$\begin{array}{l}\text { Acceptance Rate } \\
\text { (Proportion) }\end{array}$} & \multicolumn{4}{|c|}{ Confidence Rating } \\
\hline & \multicolumn{2}{|c|}{ Mixed } & \multicolumn{2}{|c|}{$\begin{array}{l}\text { Unmarked } \\
\text { Only }\end{array}$} & \multicolumn{2}{|c|}{ Mixed } & \multicolumn{2}{|c|}{$\begin{array}{l}\text { Unmarked } \\
\text { Only }\end{array}$} \\
\hline & $M$ & $S E$ & $M$ & $\overline{S E}$ & $M$ & $\overline{S E}$ & $M$ & $S E$ \\
\hline Valid believable & .89 & .02 & .93 & .02 & 1.30 & 0.06 & 1.38 & 0.06 \\
\hline Valid unbelievable & .67 & .03 & .82 & .03 & 1.10 & 0.06 & 1.17 & 0.06 \\
\hline Invalid believable & .32 & .03 & .21 & .03 & 1.02 & 0.06 & 1.14 & 0.06 \\
\hline Invalid unbelievable & .13 & .02 & .12 & .02 & 1.34 & 0.06 & 1.29 & 0.06 \\
\hline
\end{tabular}

for invalid $(M=.196, S E=.014)$ problems and for believable $(M=.59, S E=.011)$ than for unbelievable $(M=$ $.43, S E=.011)$ problems. These main effects were modified by a significant validity $\times$ markedness interaction $\left[F(1,150)=11.54, p<.001, \eta^{2}=.071\right]$ and a significant believability $\times$ markedness interaction $[F(1,150)=9.95$, $\left.p=.002, \eta^{2}=.062\right]$. The validity effect was larger in the unmarked-only $\left[F(1,75)=534.72, p<.001, \eta^{2}=.877\right]$ than in the mixed markedness $[F(1,75)=258.28, p<$ $\left..001, \eta^{2}=.775\right]$ condition. The believability effect was larger in the mixed markedness $[F(1,75)=86.56, p<$ $\left..001, \eta^{2}=.536\right]$ than in the unmarked-only $[F(1,75)=$ 20.82, $p<.001, \eta^{2}=.217$ ] condition.

The interactions were due to significantly higher acceptance of VU conclusions in the unmarked-only than in the mixed markedness condition $[F(1,150)=15.66, p<$ $\left..001, \eta^{2}=.095\right]$ and significantly higher acceptance of IB conclusions $\left[F(1,150)=6.90, p=.01, \eta^{2}=.044\right]$ in the mixed markedness than in the unmarked-only condition. Acceptance of VB and IU conclusions was not significantly affected by premise order. Thus, the conflict problems were sensitive to premise integration difficulty, but the no-conflict problems were not.

This was confirmed when accuracy was the dependent measure. A mixed 2 (validity) $\times 2$ (believability) $\times$ 2 (markedness) ANOVA revealed a significant main effect of markedness $\left[F(1,150)=11.54, p=.001, \eta^{2}=\right.$ $.071]$ and a significant validity $\times$ believability interaction $\left[F(1,150)=94.97, p<.001, \eta^{2}=.387\right]$, which were modified by a significant validity $\times$ believability $\times$ markedness interaction $\left[F(1,150)=9.95, p=.002, \eta^{2}=\right.$ $.062]$. The two-way interaction indicated that conflict (VU, IB) were more difficult than no-conflict (VB, IU) problems. This interaction was stronger in the mixed markedness $\left[F(1,75)=86.56, p<.001, \eta^{2}=.536\right]$ than in the unmarked-only $\left[F(1,75)=20.83, p<.001, \eta^{2}=\right.$ .217] condition.
The effects of markedness on belief-based and analytic processing are consistent with reasoning-first, parallelprocess, and default interventionist models for reasons similar to those described in Experiment 2.

Confidence ratings. The mean confidence ratings are shown in Table 4 . A mixed 2 (validity) $\times 2$ (believability) $\times 2$ (markedness) ANOVA revealed a significant validity $\times$ believability interaction $[F(1,150)=64.45, p<$ $\left..001, \eta^{2}=.301\right]$. As in Experiments 1 and 2, confidence was lower for conflict $(M=1.11, S E=0.04)$ than for no-conflict $(M=1.21, S E=0.03)$ problems. However, unlike in Experiments 1 and 2, in which manipulations of premise-encoding/integration difficulty affected confidence, there was no significant main effect of markedness and no interactions involving this variable in Experiment 3 [largest $F(1,150)=3.29, p=.072$ ]. One possible interpretation is that the inclusion of the unmarked-only forms facilitated premise encoding (Maybery et al., 1986) and had flow-on effects on acceptance rates but that the effect had dissipated by the time confidence ratings were made. This interpretation could be investigated in future research, but it is consistent with Shynkaruk and Thompson (2006), who found that accuracy and confidence were sensitive to different variables.

Fluid capacities and reasoning. Links with fluid intelligence and $n$-term were examined using correlations and multiple regression analyses, which are summarized in Tables 5 and 6 . Fluid intelligence was significantly positively correlated with accuracy for conflict and noconflict problems. Markedness, no-conflict accuracy, and fluid intelligence were entered as predictors in a multiple regression analysis. One participant with a large standardized residual was excluded $(N=151)$. The three predictors together accounted for $29.2 \%$ of the variance in conflict problem accuracy [multiple $R=.54 ; F(3,149)=20.19$, $p<.001]$. Markedness $(8.12 \%)$, no-conflict accuracy $(10.3 \%)$, and fluid intelligence $(3.10 \%)$ each accounted

Table 5

Zero-Order Correlations and Standard Multiple Regression of Markedness, No-Conflict Problem Accuracy, and Fluid Intelligence on Conflict Problem Accuracy $(N=151)$

\begin{tabular}{|c|c|c|c|c|c|c|c|}
\hline & Conflict Accuracy & Markedness & No-Conflict Accuracy & $B$ & $\beta$ & $s r^{2}$ (Unique) & $p$ \\
\hline Markedness & -.335 & & & -.236 & -.287 & .081 & $<<.001$ \\
\hline No-conflict accuracy & .417 & -.097 & & .503 & .336 & .103 & $<.001$ \\
\hline Fluid intelligence & .304 & -.084 & .288 & .020 & .184 & .031 & .012 \\
\hline
\end{tabular}


Table 6

Zero-Order Correlations and Standard Multiple Regression of Markedness, No-Conflict Problem Accuracy, and $n$-Term Premise Integration on Conflict Problem Accuracy $(n=58)$

\begin{tabular}{|c|c|c|c|c|c|c|c|}
\hline & $\begin{array}{l}\text { Conflict } \\
\text { Accuracy }\end{array}$ & Markedness & $\begin{array}{c}\text { No- } \\
\text { Conflict } \\
\text { Accuracy }\end{array}$ & $B$ & $\beta$ & $s r^{2}$ (Unique) & $p$ \\
\hline Markedness & -.287 & & & -.260 & -.300 & .090 & .008 \\
\hline No-conflict accuracy & .476 & .002 & & .669 & .423 & .169 & $<.001$ \\
\hline$n$-term & .308 & .055 & .240 & .020 & .223 & .047 & .053 \\
\hline
\end{tabular}

for significant independent variance, and $7.7 \%$ of the variance was shared. Thus, after controlling for markedness and no-conflict accuracy, individuals with higher fluid intelligence were better able to resolve the conflict between belief-based and analytic processes.

A similar analysis was conducted substituting $n$-term for fluid intelligence $(N=58)$. The $n$-term premise integration scores were significantly positively correlated with accuracy on conflict and no-conflict problems (Table 6). Markedness, no-conflict accuracy, and $n$-term scores together accounted for $35.6 \%$ of the variance in conflict problem accuracy [multiple $R=.597 ; F(3,54)=$ $9.96, p<.001]$. Markedness $(9 \%)$ and no-conflict accuracy $(16.9 \%)$ each accounted for significant independent variance. The unique contribution of $n$-term $(4.7 \%)$ was significant by one-tailed test ( $p=.027)$, and $5 \%$ of the variance was shared. After controlling for markedness and no-conflict accuracy, there was a trend for individuals with better integration abilities to more effectively resolve the conflict between belief-based and analytic processes.

In summary, Experiment 3 replicated the main findings of Experiments 1 and 2. Acceptance and confidence rates suggested that belief-based and analytical processes were employed in transitive inference. The participants experienced more difficulty and were less confident when these processes conflicted. Less belief-based processing and more analytic processing occurred when premise integration was facilitated by using only unmarked forms of the relational terms in the premises rather than in both marked and unmarked forms. Individuals with higher fluid intelligence and better premise integration abilities were better able to resolve conflict between analytic and belief-based processes.

\section{GENERAL DISCUSSION}

The main findings to be accounted for are the lower accuracy and lower confidence rates for conflict than for no-conflict problems, the effects of manipulations of premise integration difficulty on believability and validity effects, and the associations between the measures of fluid capacity (fluid intelligence, $n$-term) and the resolution of conflict between belief-based and analytic processes.

The three experiments suggest that belief-based and analytic processes are employed when people make transitive inferences. Significant effects of validity and believability were observed on acceptance rates in all the experiments, and problems in which belief-based and analytic processing conflicted were consistently more difficult than problems that involved no such conflict. Expressed levels of confidence showed a similar pattern. These findings are generally consistent with those in previous studies of belief bias in categorical syllogisms.

Transitive inference is more similar to relational inferences than to categorical syllogisms; therefore, the present findings will be compared with those in Roberts and Sykes (2003). Their serial presentation condition (Experiment 1) was similar to the present procedure in that premises were removed before conclusions were presented. Roberts and Sykes observed a significant main effect of validity, but there was no evidence of belief-based processing in their serial condition. They speculated that this might be due to premises and conclusions being presented separately. The present findings do not support this interpretation, because believability effects were observed with separate presentation of premises and conclusions. However, all the premises were presented at once in the present research, suggesting that sequential presentation of the premises in Roberts and Sykes's serial condition might be responsible for the difference between studies. Roberts and Sykes found significant believability effects on one-model problems in the parallel condition in which premises and conclusions were presented simultaneously. However, they were smaller in magnitude $(5 \%-6 \%)$ than those in the present experiments $(10 \%-29 \%)$. The inclu-

Table 7

Validity Effects, Believability Effects, and Confidence Levels by Condition

\begin{tabular}{|c|c|c|c|c|c|}
\hline $\begin{array}{l}\text { Premise- } \\
\text { Encoding and } \\
\text { Integration } \\
\text { Time }\end{array}$ & $\begin{array}{c}\text { Premise } \\
\text { Order }\end{array}$ & Markedness & Experiment(s) & $\begin{array}{l}\text { Validity } \\
\text { Effect }\end{array}$ & $\begin{array}{c}\text { Believability } \\
\text { Effect }\end{array}$ \\
\hline $10 \mathrm{sec}$ & Scrambled & Mixed & 1 & .35 & .29 \\
\hline $20 \mathrm{sec}$ & Scrambled & Mixed & $1,2,3$ & .59 & .19 \\
\hline $20 \mathrm{sec}$ & Consistent & Mixed & 2 & .76 & .12 \\
\hline $20 \mathrm{sec}$ & Scrambled & Unmarked only & 3 & .71 & .10 \\
\hline
\end{tabular}

Note-The baseline condition is shown in bold. 
sion of false premises in all problem types in Roberts and Sykes, but only in VU and IB problems in the present research, might account for this difference.

As was noted in the introduction, the greater difficulty and lower confidence observed for conflict than for noconflict problems in all conditions of the three experiments are consistent with reasoning-first, default interventionist, and parallel-process models. Belief-based processes involve retrieval of existing knowledge from long-term memory, whereas analytic processes including premise integration involve working memory. It is difficult to account for the greater difficulty and lower confidence levels for conflict problems without assuming that people engaged both processes.

Manipulation of premise integration difficulty had consistent effects on belief-based and analytic processing. Table 7 shows the magnitude of the validity and believability effects, expressed as mean differences in acceptance rates. The baseline condition combines the 20 -sec premise-encoding/integration (Experiment 1), the scrambled premise order (Experiment 2), and the mixed markedness (Experiment 3) conditions. Premise integration in this baseline condition is ternary relational, and this level of complexity imposes a measureable processing load in young adults (Maybery et al., 1986). Premise integration was expected to be more difficult than baseline in the 10-sec condition and easier than baseline in the consistent order and the unmarked-only conditions. Table 7 shows, as was predicted, that believability effects were larger and validity effects were smaller when premise integration was more difficult. The effects of the manipulations of premise-encoding/integration difficulty on acceptance rates and accuracy replicated previous findings (e.g., Stupple \& Ball, 2008). They supported parallelprocess and reasoning-first models more strongly than the default interventionist model, as was noted earlier.

In Experiment 3, individual differences in fluid intelligence and in premise integration ability predicted accuracy on conflict problems (VU, IB), after controlling for accuracy on nonconflict problems (VB, IU) and for markedness. This is consistent with Stanovitch and West's (2008) conclusion that the need to resolve a conflict is a key factor that determines when significant associations between fluid abilities and reasoning biases will be observed. The findings are also consistent with Quayle and Ball's (2000) finding of a larger belief bias on indeterminately invalid syllogisms for those with low working memory capacity. The observed associations are notable given the restricted range of fluid abilities among undergraduate students, as compared with the general population. These findings are consistent with three classes of dual-process models described above.

According to Sloman (1996), the demonstration of conflict effects constitutes necessary and sufficient evidence for the existence of two mental systems, each supporting one of the conflicting responses. However, Keren and Schul (2009) argued that conflicting mental states might be generated by a single system that can alternate between different modes of operation. Logical validity and believability might be two different types of external criteria that the single system uses to evaluate statements. Thus, although conflict effects are compatible with dual-process models, they are not uniquely predicted by such models. The present findings do not eliminate single-system accounts such as this.

The findings extend earlier research on categorical syllogisms and relational inference tasks by providing the first evidence of belief bias in transitive inference. The extent of belief-based versus analytic processing depended on the difficulty of premise encoding and integration, which was manipulated in three different ways. The research demonstrates the value of targeted manipulations that are based on an understanding of how participants perform the reasoning task being studied.

\section{AUTHOR NOTE}

This research was supported in part by a Griffith University Research Grant to the author. I gratefully acknowledge the assistance of the action editor, Klaus Oberauer, and three anonymous reviewers, who provided useful comments on the manuscript. Correspondence concerning this article should be addressed to G. Andrews, School of Psychology, Griffith University, Gold Coast Campus, Southport, 4222 QLD, Australia (e-mail: g.andrews@griffith.edu.au).

\section{REFERENCES}

Andrews, G., Birney, D., \& Halford, G. S. (2006). Relational processing and working memory capacity in comprehension of relative clause sentences. Memory \& Cognition, 34, 1325-1340.

AndREws, G., \& HALFORD, G. S. (1998). Children's ability to make transitive inferences: The importance of premise integration and structural complexity. Cognitive Development, 13, 479-513. doi:10.1016/ S0885-2014(98)90004-1

AndREws, G., \& HALFORD, G. S. (2002). A cognitive complexity metric applied to cognitive development. Cognitive Psychology, 45, 153-219. doi:10.1016/S0010-0285(02)00002-6

AndREws, G., \& TodD, J. M. (2008). Two sources of age-related decline in comprehension of complex relative clause sentences. In N. B. Johansen (Ed.), New research on short-term memory (pp. 93-123). New York: NovaScience.

Cattell, R. B., \& Cattell, A. K. S. (1973). Culture Fair Test of " $g$ ". Scale 2. Champaign, IL: Institute for Personality and Ability Testing.

De Neys, W., \& Van Gelder, E. (2009). Logic and belief across the lifespan: The rise and fall of belief inhibition during syllogistic reasoning. Developmental Science, 12, 123-130. doi:10.1111/j1467 $-7687.2008 .00746 . x$

Evans, J. ST. B. T. (2003). In two minds: Dual-process accounts of reasoning. Trends in Cognitive Sciences, 7, 454-459. doi:10.1016/j .tics.2003.08.012

Evans, J. ST. B. T. (2007). On the resolution of conflict in dual process theories of reasoning. Thinking \& Reasoning, 13, 321-339. doi:10.1080/1354678061008825

Evans, J. St. B. T., Barston, J. L., \& Pollard, P. (1983). On the conflict between logic and belief in syllogistic reasoning. Memory \& Cognition, 11, 295-306.

Evans, J. St. B. T., \& Curtis-Holmes, J. (2005). Rapid responding increases belief bias: Evidence for the dual-process theory of reasoning. Thinking \& Reasoning, 11, 382-389. doi:10.1080/ 1354678054200005

Evans, J. St. B. T., Newstead, S. E., \& Byrne, R. M. J. (1993). Human reasoning: The psychology of deduction. Hove, U.K.: Erlbaum.

Gilinsky, A. S., \& JudD, B. B. (1994). Working memory and bias in reasoning across the life span. Psychology \& Aging, 9, 356-371.

Goel, V., \& Dolan, R. J. (2003). Explaining modulation of reasoning by belief. Cognition, 87, B11-B22.

Halford, G. S. (1984). Can young children integrate premises in transitivity and serial order tasks? Cognitive Psychology, 16, 65-93.

Halford, G. S., Wilson, W. H., \& Phillips, S. (1998). Processing capacity defined by relational complexity: Implications for compara- 
tive, developmental, and cognitive psychology. Behavioral \& Brain Sciences, 21, 803-831.

Keren, G., \& Schul, Y. (2009). Two is not always better than one. A critical evaluation of two-system theories. Perspectives on Psychological Science, 4, 533-550.

Klauer, K. C., Musch, J., \& Naumer, R. (2000). On belief bias in syllogistic reasoning. Psychological Review, 107, 852-884.

Maybery, M. T., Bain, J. D., \& Halford, G. S. (1986). Informationprocessing demands of transitive inference. Journal of Experimental Psychology: Learning, Memory, \& Cognition, 12, 600-613.

OAKHILL, J., \& JoHNSON-LAird, P. N. (1985). The effect of belief on the spontaneous production of syllogistic conclusions. Quarterly Journal of Experimental Psychology, 37A, 553-570.

Prado, J., Van der Henst, J. B., \& Noveck, I. A. (2008). Spatial associations in relational reasoning: Evidence for a SNARC-like effect. Quarterly Journal of Experimental Psychology, 61, 1143-1150.

QuAYLE, J. D., \& BALL, L. J. (2000). Working memory, meta-cognitive uncertainty, and belief bias in syllogistic reasoning. Quarterly Journal of Experimental Psychology, 53A, 1202-1223.

RoBERTS, M. J., \& SYKES, E. D. A. (2003). Belief bias and relational reasoning. Quarterly Journal of Experimental Psychology, 56A, 131-154.

ShynKARUK, J. M., \& Thompson, V. A. (2006). Confidence and accuracy in deductive reasoning. Memory \& Cognition, 34, 619-632.

SLOMAN, S. A. (1996). The empirical case for two systems of reasoning. Psychological Bulletin, 119, 3-22.

Stanovitch, K. E., \& West, R. F. (2008). On the relative independence of thinking biases and cognitive abilities. Journal of Personality \& Social Psychology, 94, 672-695.
Stupple, E. J. N., \& Ball, L. J. (2008). Belief-logic conflict resolution in syllogistic reasoning: Inspection time evidence for a parallel process model. Thinking \& Reasoning, 14, 168-181.

Thayer, E. S., \& Collyer, C. E. (1978). The development of transitive inference: A review of recent approaches. Psychological Bulletin, 85, 1327-1343.

Thompson, V. A. (1996). Reasoning from false premises: The role of soundness in making logical deductions. Canadian Journal of Experimental Psychology, 50, 315-319.

Trabasso, T., Riley, C. A., \& Wilson, E. G. (1975). The representation of linear order and spatial strategies in reasoning: A developmental study. In R. J. Falmagne (Ed.), Reasoning: Representation and process in children and adults (pp. 201-229). Hillsdale, NJ: Erlbaum.

Viskontas, I. V., Holyoak, K. J., \& KNOWLTON, B. J. (2005). Relational integration in older adults. Thinking \& Reasoning, 11, 390-410.

Waltz, J. A., Knowlton, B. J., Holyoak, K. J., Boone, K. B., BackMadruga, C., McPherson, S., ET AL. (2004). Relational integration and executive function in Alzheimer's disease. Neuropsychology, 18, 296-305.

Waltz, J. A., Knowlton, B. J., Holyoak, K. J., Boone, K. B., MishKin, F. S., De Menezes Santos, M., ET AL. (1999). A system for relational reasoning in human prefrontal cortex. Psychological Science, 10, 119-125.

(Manuscript received September 18, 2009; revision accepted for publication March 29, 2010.) 